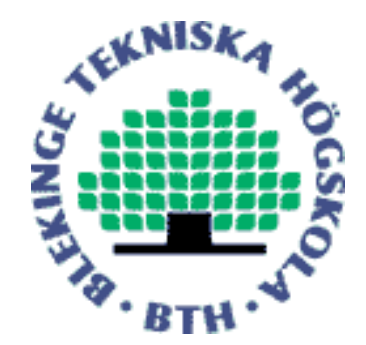

Copyright @ 2010 IEEE.

Citation for the published paper:

Title:

In press: RFI Suppression in Ultrawideband SAR Using an Adaptive Line Enhancer

Author:

Viet Thuy Vu, Thomas Sjögren, Mats Pettersson, Lars Håkansson, Anders Gustavsson, Lars Ulander

Journal:

Geoscience and Remote Sensing Letters

Year:

2010

Vol:

7

Issue:

4

Pagination:

694-698

URL/DOI to the paper:

10.1109/LGRS.2010.2045633

This material is posted here with permission of the IEEE. Such permission of the IEEE does not in any way imply IEEE endorsement of any of BTH's products or services Internal or personal use of this material is permitted. However, permission to reprint/republish this material for advertising or promotional purposes or for creating new collective works for resale or redistribution must be obtained from the IEEE by sending a blank email message to pubs-permissions@ieee.org.

By choosing to view this document, you agree to all provisions of the copyright laws protecting it. 


\title{
RFI Suppression in Ultrawideband SAR Using an Adaptive Line Enhancer
}

\author{
Viet T. Vu, Student Member, IEEE, Thomas K. Sjögren, Student Member, IEEE, Mats I. Pettersson, Member, IEEE, \\ Lars Håkansson, Anders Gustavsson, and Lars M. H. Ulander, Senior Member, IEEE
}

\begin{abstract}
In this letter, we propose an approach to suppress radio-frequency interference (RFI) in ultrawideband (UWB) low-frequency synthetic aperture radar (SAR). According to the proposal, RFI is suppressed by using an adaptive line enhancer controlled by the normalized least mean square algorithm. The approach is tested successfully on real UWB low-frequency SAR data. In order to keep the computational burden down, possible ways to integrate the RFI suppression approach into SAR imaging algorithms are also suggested.
\end{abstract}

Index Terms-Adaptive line enhancer (ALE), CARABAS-II, normalized least mean square (LMS) (NLMS), radio-frequency interference (RFI), synthetic aperture radar (SAR), ultrawideband (UWB).

\section{INTRODUCTION}

$\mathbf{P}$ RACTICAL issues in ultrawideband (UWB) synthetic aperture radar (SAR) data processing have been described in [1]. Among these, radio-frequency interference (RFI) suppression has not attracted much attention in recent publications. This may be explained by the dependence of RFI on the operating frequency of the SAR systems. An early approach, which adaptively filters RFI in a simulated baseband SAR signal (40 MHz), is introduced in [2]. Another approach in the frequency domain with amplitude normalization aiming at low-frequency UWB SAR systems is presented in [3]. The limitations of these approaches can mainly be found in the least mean square (LMS) adaptive filter, which may be sensitive to nonstationary SAR scenes. In addition, the results of RFI suppression obtained with these approaches are demonstrated mainly in relation to the raw radar signal. The evaluation of their performance may be insufficient. For example, the efficiency of RFI suppression in the approaches presented in [2] and [3] is not clearly defined or is not compared with other methods. The computational burden demanded by these approaches is not discussed, and no solution to reduce the computational complexity is suggested. There are also other approaches such as notch filtering during the image correlation process reserved for Japanese Earth Resources Satellite 1 SAR

Manuscript received December 16, 2009. Date of publication May 10, 2010; date of current version October 13, 2010.

V. T. Vu, T. K. Sjögren, M. I. Pettersson, and L. Håkansson are with the Blekinge Institute of Technology, 37179 Karlskrona, Sweden (e-mail: viet.thuy.vu@bth.se; thomas.sjogren@bth.se; mats.pettersson@bth.se; lars. hakansson@bth.se).

A. Gustavsson and L. M. H. Ulander are with the Swedish Defence Research Agency, 58111 Linköping, Sweden (e-mail: anders.gustavsson@ foi.se; lars.ulander@foi.se).

Digital Object Identifier 10.1109/LGRS.2010.2045633

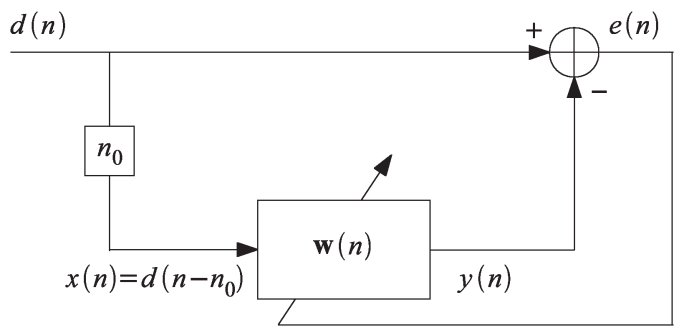

Fig. 1. ALE structure.

[4] and the filtering approach for synthesized SAR images proposed in [5] and tested with E-SAR L-band data.

In this letter, we propose to use an adaptive line enhancer (ALE) [6] controlled by the Normalized LMS (NLMS) algorithm to eliminate RFI. To evaluate the performance of the proposed method, data collected by an airborne UWB low-frequency SAR system, CARABAS-II [7], have been used. The collected data comprise a point target—a corner reflector-deployed in the SAR scene. Moreover, the proposed RFI suppression approach is compared with the Linear RFI Filtering (LRF) technique [8] which is currently used in CARABAS-II data processing. Also, the computational cost of the ALE in this application has been addressed, and a strategy for integrating it into the SAR imaging algorithms is recommended.

\section{RFI SUPPRESSION BY ALE}

ALE is essentially adaptive noise cancellation without a separate reference signal. In ALE, the input signal is delayed to generate the reference signal. Fig. 1 shows the ALE structure. If the radar echo is used as input to the ALE, the input $d(n)$ is defined by

$$
d(n)=s(n)+\nu(n)+\rho(n)
$$

where $s(n)$ is the reflected UWB radar signal, i.e., the chirp signal, $\nu(n)$ is the thermal noise signal, and $\rho(n)$ is the RFI signal. Furthermore, $x(n)$ is the reference signal to the adaptive finite-impulse response (FIR) filter $\mathbf{w}(n)$, and this is a delayed version of the input signal, i.e., $x(n)=d\left(n-n_{0}\right)$, where $n_{0}$ is the delay in sample. If the adaptive FIR filter has the order $L$, its coefficient vector $\mathbf{w}(n)$ and reference signal vector $\mathbf{x}(n)$ are given by

$$
\begin{aligned}
\mathbf{w}(n) & =\left[\begin{array}{llll}
w_{0}(n) & w_{1}(n) & \cdots & w_{L-1}(n)
\end{array}\right]^{\mathrm{T}} \\
\mathbf{x}(n) & =\left[\begin{array}{llll}
x(n) & x(n-1) & \cdots & x(n-L+1)
\end{array}\right]^{\mathrm{T}}
\end{aligned}
$$


respectively. Assuming that the input signal $d(n)$ is a weakly stationary real stochastic process, the cross correlation between the input signal $d(n)$ and the reference signal $x(n)$ is given by

$$
r_{d x}(k)=E[d(n) x(n-k)], \quad k \in Z .
$$

By using the fact that the RFI signal is a narrowband signal and selecting the minimum delay $n_{0}$ that decorrelates the UWB radar and the thermal noise signals in the radar echo $d(n)$ and in the reference signal $x(n)$, the cross correlation can be shown to be

$$
\begin{aligned}
r_{d x}(k) & =E[d(n) x(n-k)] \\
& =E\left[\rho(n) \rho\left(n-n_{0}-k\right)\right], \quad k \geq 0 .
\end{aligned}
$$

If it is assumed that the ALE FIR filter has converged to its Wiener filter coefficients, the output signal of the filter will thus be an estimate of the RFI signal

$$
\rho(n) \approx y(n)=\mathbf{w}^{\mathrm{T}}(n) \mathbf{x}(n) .
$$

Hence, the estimation error signal $e(n)$ will be an estimate of the UWB radar signal plus the thermal noise signal

$$
e(n)=d(n)-y(n) \approx s(n)+\nu(n) .
$$

An airborne UWB SAR system is usually associated with a long and nonlinear sensor trajectory. The high energy radiating from RFI sources can appear anywhere and anytime in an illuminated ground scene. To handle the nonstationary ground scene, and thus a nonstationary reference signal to the adaptive filter, the NLMS algorithm is proposed to adjust the adaptive filter coefficients

$$
\mathbf{w}(n+1)=\mathbf{w}(n)+\frac{\beta}{\mathbf{x}^{\mathrm{T}}(n) \mathbf{x}(n)+\epsilon} \mathbf{x}^{*}(n) e(n)
$$

where $0<\beta<2$ and $\epsilon$ is a small positive real number. The starting set of the filter coefficient vector $\mathbf{w}(n)$ can be selected as

$$
\mathbf{w}(0)=\left[\begin{array}{llll}
0 & 0 & \cdots & 0
\end{array}\right]^{\mathrm{T}} .
$$

\section{EXPERIMENTAL Results AND Evaluation}

In this section, some experimental results are presented to illustrate and evaluate the approach proposed in the previous section. The CARABAS-II [7] data are used in the experiments. CARABAS-II operates in the lower very high frequency (VHF) band from 20 to $90 \mathrm{MHz}$. This means that the radar system operates in an environment with a high thermal noise level and frequency bands with very strong broadcasters. The influence of these broadcasters is considered to be extremely significant since their radiated power is much higher than the reflected power of CARABAS-II.

Fig. 2(a) shows the imaged ground scene of the island of Visingsö located in southern Sweden using data collected by the CARABAS-II system. As a part of imaging the ground scene, a corner reflector was deployed in the center of the ground scene. This corner reflector should appear as a point target in the area

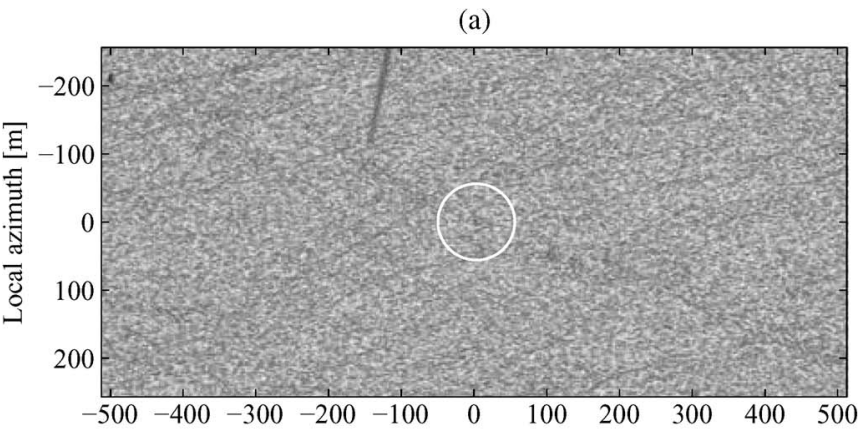

(b)

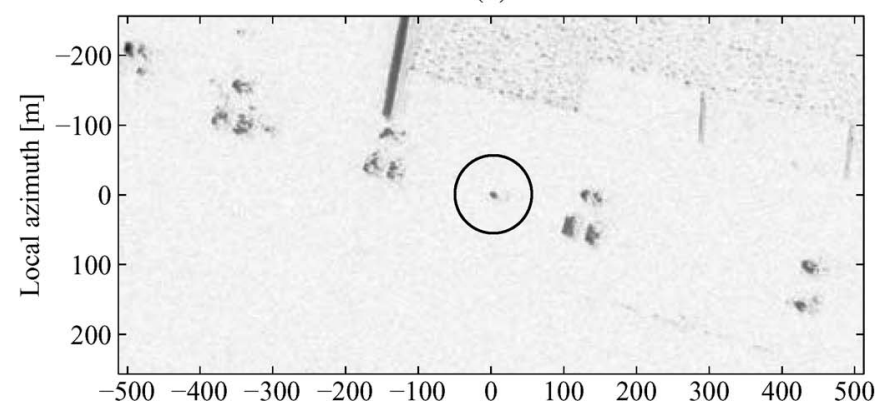

(c)

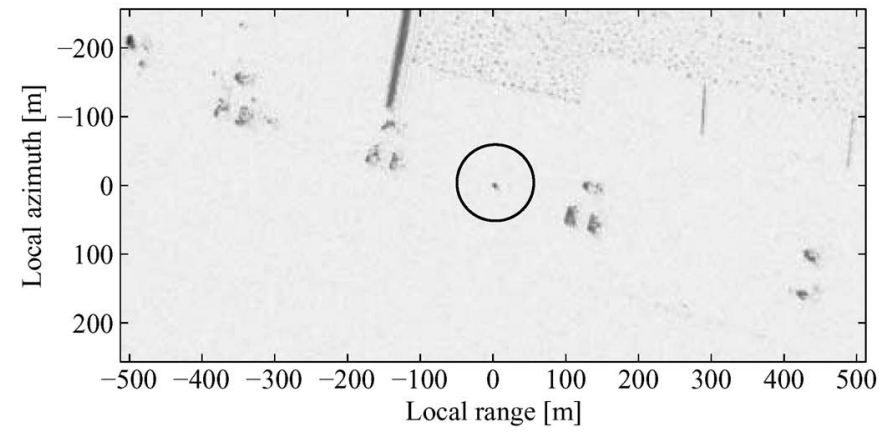

Fig. 2. SAR images of the illuminated ground scene. The white/black circles in the SAR images mark the position of the corner reflector which has been deployed in the field campaign. (a) SAR image formed with the original radar echo containing the RFI signal. (b) SAR image formed with the estimated signal using ALE. (c) SAR image formed with the estimated signal using LRF.

marked by the white circle in Fig. 2(a). However, the point target is totally hidden by RFI, and only very strong scatterers on the island can be seen in the SAR image. The influence of RFI on the SAR images has been shown to be affected by the integration angle [9]. However, this will not be considered in this letter, and the complete aperture, i.e., the widest possible integration angle, is used.

The original range-compressed radar echo containing the RFI signal and the magnitude of its discrete Fourier transform (DFT) are shown in Fig. 3(a) and (b), respectively. The number of range samples is $N=16384$. RFI, e.g., at the frequency of about $27 \mathrm{MHz}$, and others in the range from 48 to $68 \mathrm{MHz}$, can be observed in Fig. 3(b). Such RFI may be caused by the Citizens' band (26.965-27.405 MHz) and television channels 2-6 (54-88 MHz). Fig. 3(b) also shows the extremely high magnitude of the RFI component (up to $100 \mathrm{~dB}$ ) compared with the magnitude of the flat UWB radar spectrum (only about $60 \mathrm{~dB}$ ). This also means that the RFI signal dominates in the radar echo. A more illustrative analysis can be found in [9], 
(a)

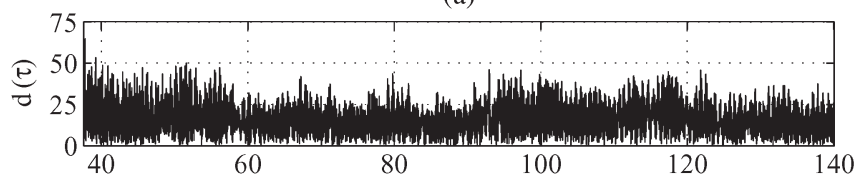

(c)

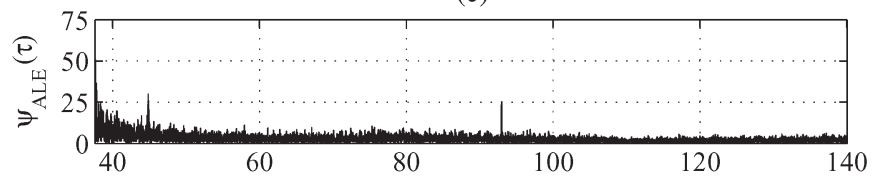

(e)

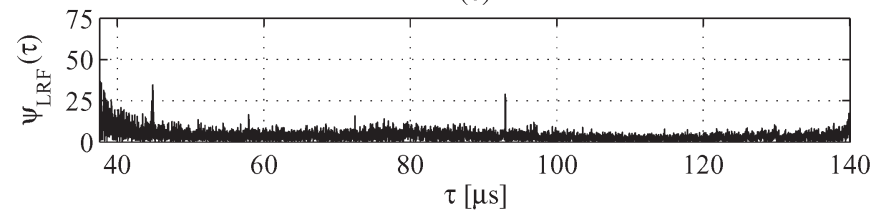

(b)

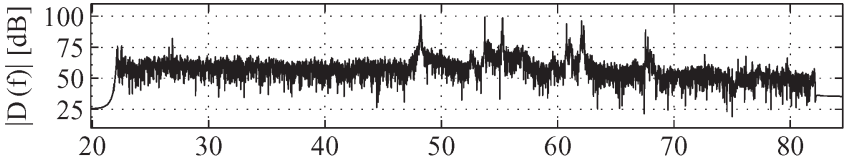

(d)

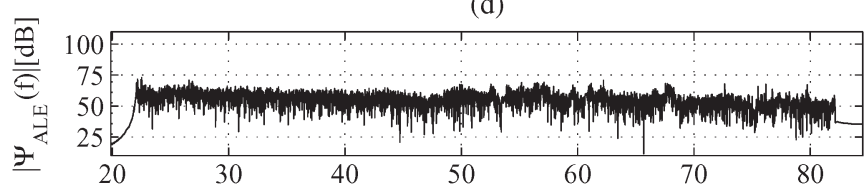

(f)

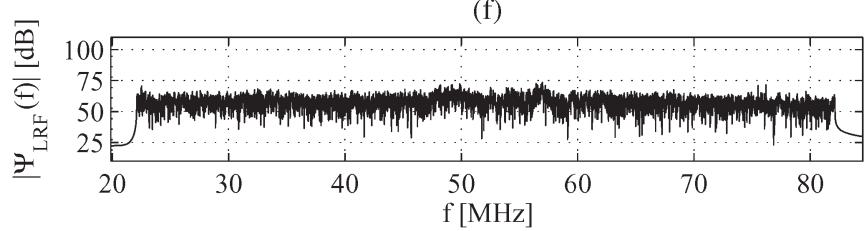

Fig. 3. Range-compressed radar echo containing the RFI signal, the estimated signal using ALE, the estimated signal using LRF in the time domain, and the magnitudes of their DFT in the frequency domain, i.e., their periodograms. The unit of the radar echo and the estimated signals are given in the dimensionless scale, and the magnitudes of their DFT are in the decibel scale. In the plots, the time axis $\tau$ instead of the sample axis $n$ is used. (a) Radar echo containing the RFI signal and (b) the magnitude of its DFT. (c) Estimated signal using ALE and (d) the magnitude of its DFT. (e) Estimated signal using LRF and (f) the magnitude of its DFT.

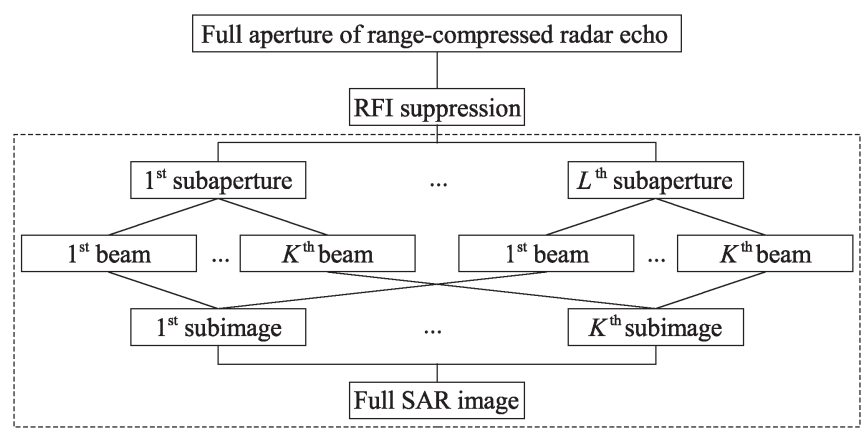

Fig. 4. Proposed RFI suppression scheme. The time-domain LBP algorithm is bounded by the dashed-line rectangle.

where the radar echo is simulated according to CARABAS-II parameters. The RFI suppression scheme is shown in Fig. 4. According to this scheme, the original range-compressed radar echo containing the RFI signal is first processed by ALE and then by the Local Backprojection algorithm (LBP) [10]. This algorithm will be presented in detail in Section IV where a possibility to integrate the RFI suppression approach into SAR imaging algorithms is suggested. In the following RFI suppression experiment, an adaptive filter of the order $L=1024$ has been used in the ALE and the delay set to $n_{0}=1$. The selection of $\beta$ is critical for the performance of the NLMS when using an ALE [6]. In this letter, the step size of NLMS is experimentally selected by $\beta=0.1$. The estimated radar signal plus the thermal noise signal is given to $e(n)$ in Fig. 1 and is shown in Fig. 3(c). In the context of this letter, the estimated radar signal plus the thermal noise signal using the proposed RFI suppression approach is called the estimated signal using ALE and denoted by $\psi_{\mathrm{ALE}}$ in the plots. Its amplitude is significantly lower than the amplitude of the radar echo containing the RFI signal, which is shown in Fig. 3(a). The magnitude of the DFT of the estimated signal using ALE is shown in Fig. 3(d) and is substantially flatter as compared with the magnitude of the DFT of the radar echo containing the RFI signal shown in Fig. 3(b). A new SAR image is formed by the estimated signal using ALE and is shown in Fig. 2(b). The ground scene, in general, and the corner reflector, in particular, can now be observed.

A basic evaluation of the proposed RFI suppression approach is facilitated by comparing it with the LRF technique [8] which is currently used in CARABAS-II data processing. The linear filter in LRF is defined by inverting the averaged range frequency response. This technique has been proven to be robust, nonadaptive over one aperture, nonsupportive for real-time processing, and unable to handle short-time RFI. The estimated radar signal using this technique is called the estimated signal using LRF and denoted by $\psi_{\mathrm{LRF}}$ in the plots. The estimated signal using LRF and the magnitude of its DFT are shown in Fig. 3(d) and (e), respectively. The estimated signal using ALE can be visually evaluated to be almost identical to the estimated signal using LRF. In the frequency domain, the flatness of the magnitude of the DFT of the estimated signal using ALE is close to the one using LRF. However, using ALE to suppress RFI may cause frequency attenuations at the RFI frequencies. This is demonstrated in the experiments on the simulated data given in [9]. There is no clear difference between the SAR image formed with the estimated signal using ALE, shown in Fig. 2(b), and the SAR image formed with the estimated signal using LRF, shown in Fig. 2(c).

This situation can be further evaluated by SAR image-quality assessments. These assessments are routinely made with the help of corner reflectors which are widely used for calibration in field campaigns [11]. In the considered SAR scene, SAR image-quality assessments are possible due to the corner reflector deployed in the center of the SAR scene, appearing as a point target in the SAR images. The signal-to-interferencenoise ratio (SINR) is estimated by the ratio of the peak intensity of the point target to the average intensity in the area $(-512$ : $-256,0: 256)$ of the SAR images. In this area, backscattering from the surrounding clutter is low. The results, which are 
TABLE I

MeAsured Results on the Point TARget

\begin{tabular}{lcccc}
\hline \hline & SINR & $\Delta_{r}$ & ISLR & PSLR \\
\hline ALE IR-SAR & $\approx 27 \mathrm{~dB}$ & $\approx 2.8 \mathrm{~m}$ & $\approx-40 \mathrm{~dB}$ & $\approx-20 \mathrm{~dB}$ \\
LRF IR-SAR & $\approx 28 \mathrm{~dB}$ & $\approx 3.0 \mathrm{~m}$ & $\approx-33 \mathrm{~dB}$ & $\approx-17 \mathrm{~dB}$ \\
\hline
\end{tabular}

summarized in Table I, show that SINR measured on the SAR image formed with the estimated signal using ALE is $1 \mathrm{~dB}$ lower in comparison with the one using LRF. This can be interpreted as the estimated error caused by ALE.

At the peak intensity of the point target in the SAR images, vectors are extracted in the range direction. The reason for this extraction is explained by the greater sensitivity of the sidelobes in the range direction compared with that in the azimuth direction produced by UWB SAR systems [12]. These vectors are interpreted as measured Impulse Responses in SAR imaging (IR-SAR) [13] in the range direction of the point target. They are normalized with respect to the peak intensity of the point target and then plotted in the same figure for comparison purposes. As shown in Fig. 5, the measured IR-SAR extracted from the SAR image formed with the radar echo containing the RFI signal, which is briefly called as original IR-SAR in this letter, is seriously distorted. For this reason, the point target is difficult to see in this SAR image. Some differences between the measured IR-SAR, which are extracted from the SAR image formed with the estimated signal using ALE, and the measured IR-SAR extracted from the SAR image formed with the estimated signal using LRF, can also be observed in Fig. 5. These differences may be generated from the nonadaptive (average) and adaptive characteristics of the two approaches. The measured results from these IR-SAR, stated as range resolution, integrated sidelobe ratio and peak sidelobe ratio, are also summarized in Table I. They show that, in this specific case, the performance of the proposed approach is similar to that of the LRF technique.

According to (7), the performance of ALE depends strongly on the ratio of the ability to decorrelate the UWB radar signal in the radar echo $d(n)$ and in the reference signal $x(n)$. In other words, it depends on the interrelation of the UWB radar signal bandwidth and the RFI signal bandwidth. In environments where there are RFI with bandwidths so large that they might also be decorrelated by the minimum delay selection $n_{0}$, the ALE performance is likely to decrease.

\section{Possibility to Integrate the RFI SUPPRESSION APPROACH INTO SAR IMAGING ALGORITHMS}

In this section, we discuss a possibility to integrate the proposed RFI suppression approach into SAR imaging algorithms. The background of this discussion is due to the computational burden required by the proposed RFI suppression. The experimental results indicate that the proposed approach using ALE allows for adaptive and efficient RFI suppression in terms of quality. However, this process may be inefficient in terms of processing time. In order to keep the computational burden down, this letter also suggests integrating the RFI suppression approach into SAR imaging algorithms. The discussion will

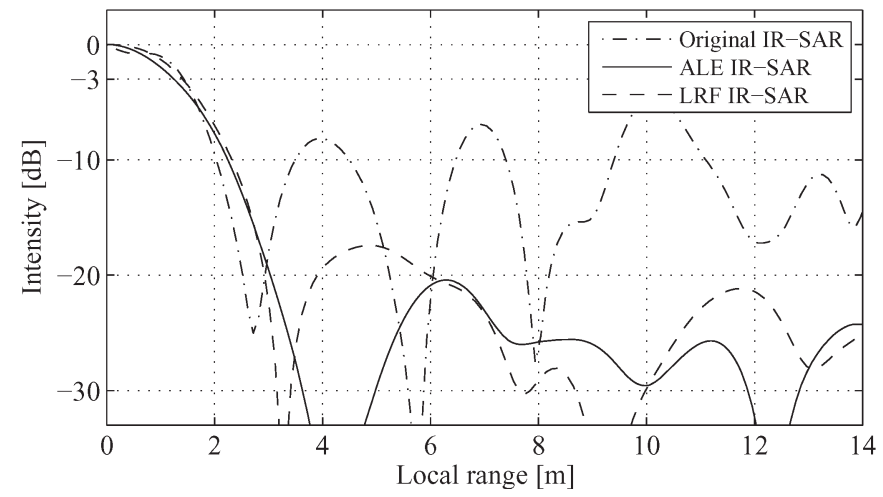

Fig. 5. IR-SAR of the point target extracted in the range direction from the SAR images formed with the radar echo containing the RFI signal, the estimated signal using ALE, and the estimated signal using LRF.

focus on time-domain algorithms which are recommended for UWB SAR data processing [14].

In the SAR technique, a time-domain backprojection algorithm refers to a superposition of the backprojected radar echo. To save processing time, it is recommended to process the radar echo on a subaperture subimage basis and in multiple beamforming stages [15], i.e., fast factorized backprojection. The integration of the RFI suppression approach into any beamforming stage helps to reduce the number of operations.

We consider here a two-stage SAR imaging algorithm, i.e., LBP [10]. In LBP, the complete aperture and the full SAR image are segmented in $M$ subapertures and $K$ subimages, respectively. The reconstruction of the imaged scene is therefore split into two stages, i.e., beam-forming and backprojection. In the beam-forming stage, from the center of the $m$ th subaperture $x_{m}$, the $k$ th beam, aiming at the center of the $k$ th subimage, is formed by a superposition of all radar echo data in the subaperture. The $k$ th subimage is then backprojected by the $k$ th beam. This procedure is repeated for all subapertures. The full SAR image is retrieved by a coherent combination of all subimages. The RFI suppression approach can be integrated into LBP between the beam-forming and backprojection stages. With such, the number of operations required by the approach is reduced by

$$
\frac{N_{\text {sub-a. }} \times N_{\text {sub-b. }}}{N_{\text {sub- } i .}}
$$

where $N_{\mathrm{sub}-a}$. and $N_{\mathrm{sub}-i \text {. are }}$ the number of the aperture positions in one subaperture and the number of subimages, respectively. The ratio of the original radar-echo sample to the beam sample is denoted by $N_{\text {sub-b. }}$. The proposed scheme for this integration is shown in Fig. 6. Fig. 7 shows an example of an estimated beam using ALE. In this example, the beam is formed with a subaperture of $N_{\text {sub-a. }}=32$ aperture positions and the number of subimages is $N_{\mathrm{sub}-i}=4$. For illustration purposes, we use the ratio of the original radar-echo sample to the beam sample $N_{\text {sub-b. }}=1$. The amplitude of the estimated beam is shown in Fig. 7(a), and the magnitude of its DFT is shown in Fig. 7(b). The magnitude of its DFT can be evaluated as moderately flat. However, in this example, the number of operations required to run the approach is reduced by a factor of eight. The RFI suppressed beams can then be used in the backprojection stage to form a SAR image. 


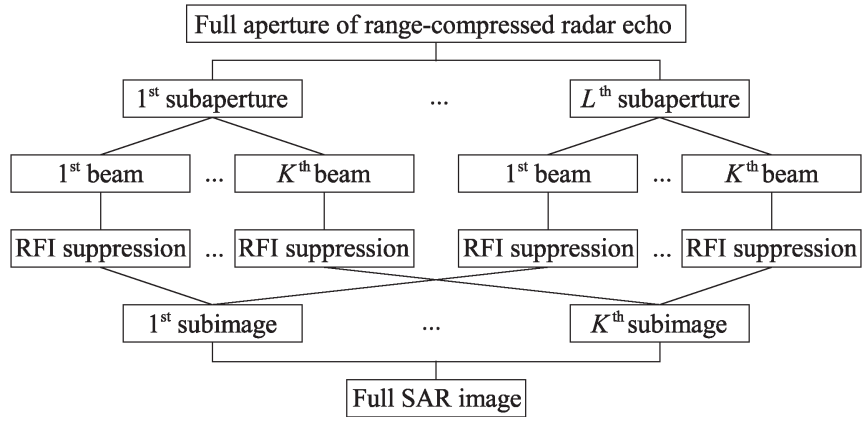

Fig. 6. Scheme to integrate the RFI suppression approach into LBP.

(a)

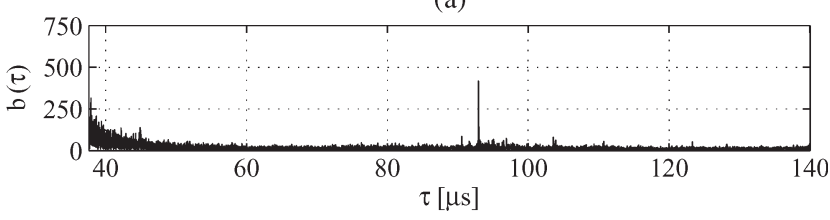

(b)

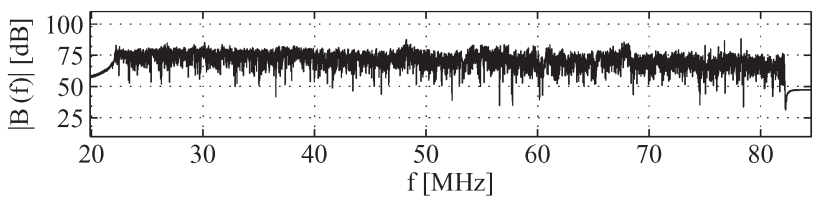

Fig. 7. (a) Estimated beam using ALE and (b) the magnitude of its DFT. The original beam is formed by 32 original radar echoes containing the RFI signal.

\section{CONCLUSION}

In this letter, an approach to suppress RFI in UWB lowfrequency SAR is proposed. The approach is based on the ALE mechanism controlled by NLMS. The approach is tested successfully on the CARABAS-II data. The possibility to integrate this RFI suppression approach into SAR imaging algorithms in order to obtain efficiency in processing time is also discussed.

\section{ACKNOWLEDGMENT}

The authors would like to thank the KK Foundation for making this research project possible and the Swedish Defence
Research Agency, Saab Bofors Dynamics, Saab Microwave Systems, and RUAG Aerospace for their support.

\section{REFERENCES}

[1] R. Goodman, S. Tummala, and W. Carrara, "Issues in ultra-wideband, widebeam SAR image formation," in Proc. IEEE Int. Radar Conf., Alexandria, VA, May 1995, pp. 479-485.

[2] C. T. Le, S. Hensley, and E. Chapin, "Adaptive filtering of RFI in wideband SAR signals," in Proc. 7th Annu. JPL Airborne Earth Sci. Workshop, Pasadena, CA, Jan. 1998.

[3] X. Luo, L. M. H. Ulander, J. Askne, G. Smith, and P.-O. Frolind, "RFI suppression in ultra-wideband SAR systems using LMS filters infrequency domain," Electron. Lett., vol. 37, no. 4, pp. 241-243, Feb. 2001.

[4] H. Kimura, T. Nakamura, and K. P. Papathanassiou, "Suppression of ground radar interference in JERS-1 SAR data," IEICE Trans. Commun., vol. 87, no. 12, pp. 3759-3765, 2004.

[5] A. Reigber and L. Ferro-Famil, "Interference suppression in synthesized SAR images," IEEE Geosci. Remote Sens. Lett., vol. 2, no. 1, pp. 45-49, Jan. 2005.

[6] M. H. Hayes, Statistical Digital Signal Processing and Modeling. New York: Wiley, 1996.

[7] A. Gustavsson, L. M. H. Ulander, B. H. Flood, P.-O. Frölind, H. Hellsten, T. Jonsson, B. Larsson, and G. Stenstrom, "Development and operation of an airborne VHF SAR system-lessons learned," in Proc. IEEE IGARSS, Seattle, WA, Jul. 1998, vol. 1, pp. 458-462.

[8] L. M. H. Ulander and P.-O. Frölind, "Precision processing of CARABAS HF/VHF-band SAR data," in Proc. IEEE IGARSS, Hamburg, Germany, Jun. 1999, vol. 1, pp. 47-49.

[9] V. T. Vu, T. K. Sjögren, M. I. Pettersson, and L. Håkansson, "An approach to suppress RF interference in ultrawideband low frequency synthetic aperture radar," in Proc. IEEE Radar, Washington, DC, 2010, pp. 1381-1385.

[10] O. Seger, M. Herberthson, and H. Hellsten, "Real time SAR processing of low frequency ultra wide band radar data," in Proc. EUSAR, Friedrichshafen, Germany, May 1998, pp. 489-492.

[11] M. Shimada, O. Isoguchi, T. Tadono, and K. Isono, "PALSAR radiometric and geometric calibration," IEEE Trans. Geosci. Remote Sens., vol. 47, no. 12, pp. 3915-3932, Dec. 2009.

[12] V. T. Vu, T. K. Sjögren, M. I. Pettersson, and A. Gustavsson, "Definition on SAR image quality measurements for UWB SAR," in Proc. SPIE Image Signal Process. Remote Sensing XIV, Cardiff, U.K., Sep. 2008, vol. 7109, pp. 710 91A-1-71091A-9.

[13] V. T. Vu, T. K. Sjögren, M. I. Pettersson, and H. Hellsten, "An impulse response function for evaluation of UWB SAR imaging," IEEE Trans. Signal Process., vol. 58, no. 7, pp. 3927-3932, Jul. 2010.

[14] V. T. Vu, T. K. Sjögren, and M. I. Pettersson, "A comparison between fast factorized backprojection and frequency-domain algorithms in UWB low frequency SAR," in Proc. IEEE IGARSS, Boston, MA, Jul. 2008, pp. 1293-1296.

[15] L. M. H. Ulander, H. Hellsten, and G. Stenstrom, "Synthetic-aperture radar processing using fast factorized back-projection," IEEE Trans. Aerosp. Electron. Syst., vol. 39, no. 3, pp. 760-776, Jul. 2003. 